\title{
New protocol for construction of eyeglasses-supported provisional nasal prosthesis using CAD/CAM techniques
}

\author{
Leonardo Ciocca, DDS, PhD; ${ }^{\text {* }}$ Massimiliano Fantini, MechE, PhD; ${ }^{2}$ Francesca De Crescenzio, IE, PhD; ${ }^{2}$ \\ Franco Persiani, Eng, PhD; ${ }^{2}$ Roberto Scotti, MD, DDS ${ }^{1}$ \\ ${ }^{1}$ Department of Oral Sciences, University of Bologna, Bologna, Italy; ${ }^{2}$ II Faculty of Engineering, University of Bologna, \\ Forli, Italy
}

\begin{abstract}
A new protocol for making an immediate provisional eyeglasses-supported nasal prosthesis is presented that uses laser scanning, computer-aided design/computer-aided manufacturing procedures, and rapid prototyping techniques, reducing time and costs while increasing the quality of the final product. With this protocol, the eyeglasses were digitized, and the relative position of the nasal prosthesis was planned and evaluated in a virtual environment without any try-in appointment. This innovative method saves time, reduces costs, and restores the patient's aesthetic appearance after a disfiguration caused by ablation of the nasal pyramid better than conventional restoration methods. Moreover, the digital model of the designed nasal epithesis can be used to develop a definitive prosthesis anchored to osseointegrated craniofacial implants.
\end{abstract}

Key words: CAD/CAM, computer-aided design, facial disfigurement, facial rehabilitation, immediate temporary prosthesis, maxillofacial rehabilitation, nasal prosthesis rapid prototyping, reverse engineering, silicone prosthesis, virtual molding.

\section{INTRODUCTION}

After tumor removal or accidental injury, such as a gunshot or road accident, the plastic surgeon should do whatever possible using skin and cartilage to restore a defect involving the nose. Sometimes, however, the patient's condition does not allow plastic surgery rehabilitation, necessitating a silicone nasal prosthesis, which provides good aesthetics, respiratory function, and social relationship recovery.
The methods most commonly used for retaining nasal prostheses include medical adhesives, mechanical support (e.g., eyeglasses), and osseointegrated craniofacial implants [1-6]. The medical adhesives used to retain prostheses may irritate soft tissues that contact the prosthesis and may damage the thin margins of the prosthesis when it is removed for maintenance and cleaning. An eyeglasses-supported nasal prosthesis system may result in displacement, with an opening at the margins of the prosthesis. For definitive rehabilitation, osseointegrated craniofacial implants (two positioned in the premaxilla in the nasal floor and one in the glabella, if necessary) and a metal support bar to stabilize the prosthesis are best for anchoring a nasal prosthesis to the skull.

Because manufacturing a definitive implant-supported nasal prosthesis takes a long time and the patient needs to have an acceptable aesthetic appearance as soon as possible, a provisional eyeglasses-supported nasal prosthesis may be a temporary solution. Moreover, the use of eyeglasses to support the nasal prosthesis may be necessary when insufficient bone exists for implant placement.

Abbreviations: 3-D $=$ three-dimensional, $\mathrm{ABS}=$ acrylonitrile butadiene styrene, $\mathrm{CAD} / \mathrm{CAM}=$ computer-aided design/computer-aided manufacturing, FDM = fused deposition modeling.

*Address all correspondence to Leonardo Ciocca, DDS, PhD; University of Bologna, Department of Oral Sciences, S. Vitale 59, Bologna 40126, Italy; +39-051-2088145; fax: +39-051-220852. Email: leonardo.ciocca@unibo.it

DOI:10.1682/JRRD.2009.11.0189 
Recently, several authors have attempted to design facial prostheses (auricular and nasal) using computeraided design/computer-aided manufacturing (CAD/CAM) technology to reduce time and costs and to increase the quality of the final product [7-13].

In 2007 and 2009, protocols for provisional eyeglassessupported nasal prostheses were published [14-16]. These studies developed a simple transfer system requiring try-in on the patient's face for correct positioning of the eyeglasses and prosthesis with the use of a manual siliconebased approach. The investigators placed eyeglasses on the patient's face and used silicone to check the relationship with the nasal prosthesis wax-up to design the substructure framework used to connect the prosthesis to the eyeglasses.

This article presents an update of this method in which the eyeglasses were digitized and the relative position of the nasal prosthesis was planned and evaluated in a virtual environment without any try-in appointment, further reducing the delivery time and patient discomfort.

\section{MATERIALS AND METHODS}

A 58-year-old man lost his entire nose after being shot (Figure 1). He was scheduled for a definitive nasal prosthesis anchored to osseointegrated craniofacial implants. His general medical condition, which did not allow surgical reconstruction, determined the schedule. In the meantime, to

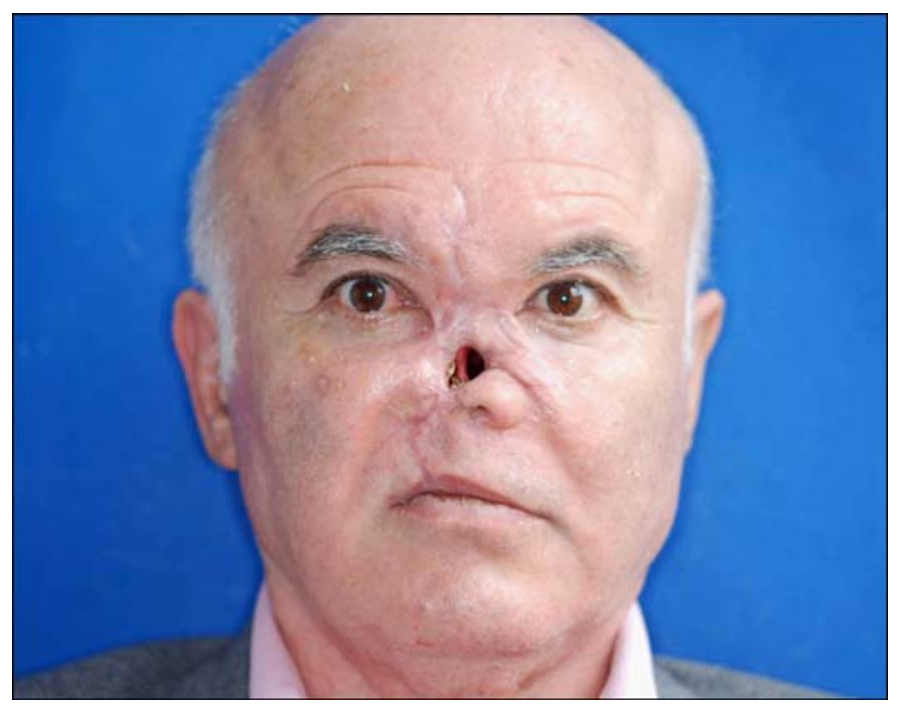

Figure 1.

Initial appearance of patient's face with injury from accidental gunshot. provide an immediate temporary solution and to enable the patient to recover socially, the maxillofacial prosthodontist constructed an eyeglasses-supported provisional prosthesis.

The flow chart of the process used to make the provisional prosthesis is shown in Figure 2. First, the patient's facial defect and surrounding anatomical structures, including both ears for later positioning of the digital model of the eyeglasses, were scanned with a three-dimensional (3-D) laser. We used a laser scanner (NextEngine Desktop 3-D Scanner; NextEngine, Inc; Santa Monica, California) to obtain a digital model of the entire face while the patient's eyes were covered with a protective anti-ultraviolet device, although the class $1 \mathrm{M}$ laser beam is classified as eye-safe scanning (Figure 3). The face was scanned from five (left, right, frontal, upper, and lower) different perspectives around the patient's head to detect all undercuts. With the face visualized as a clock, the "upper" position was at 12 hours, and the "lower" at 6 hours, while left and right positions were referred to as the lateral profiles. During 3-D

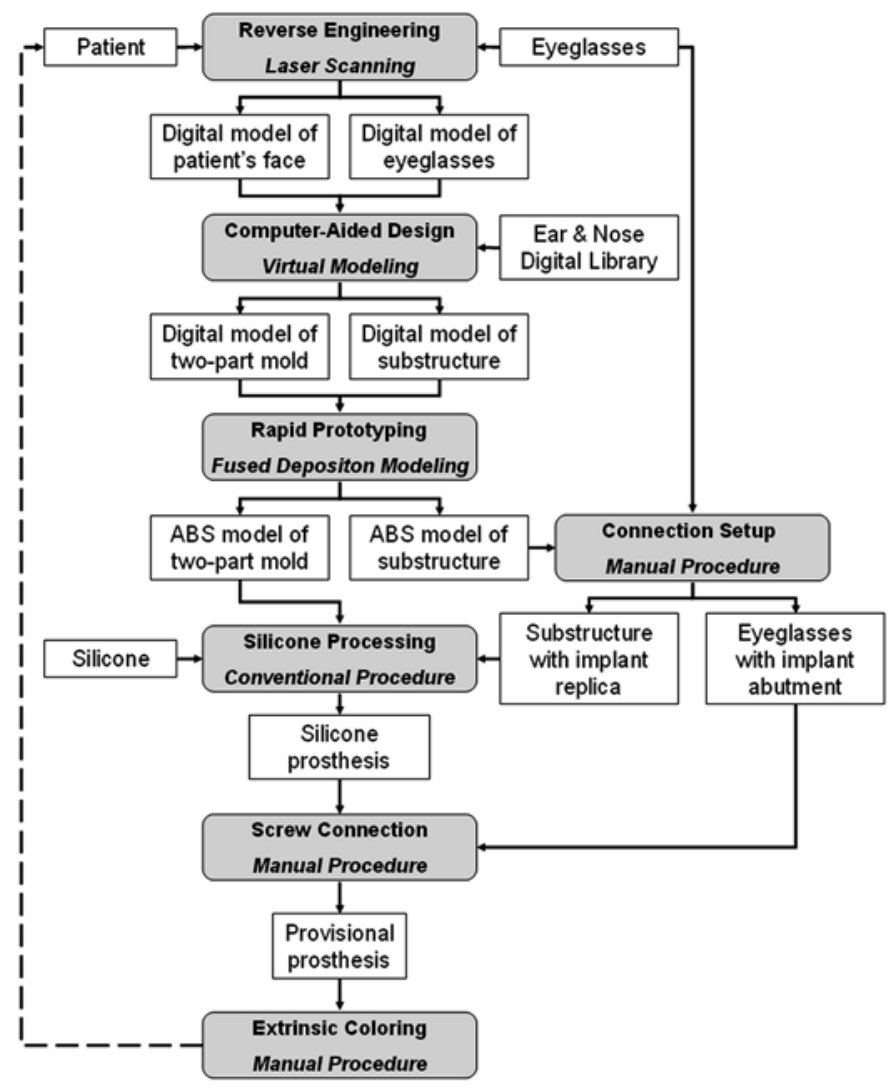

Figure 2.

Flow chart of procedure for provisional nasal prosthesis. ABS = acrylonitrile butadiene styrene. 


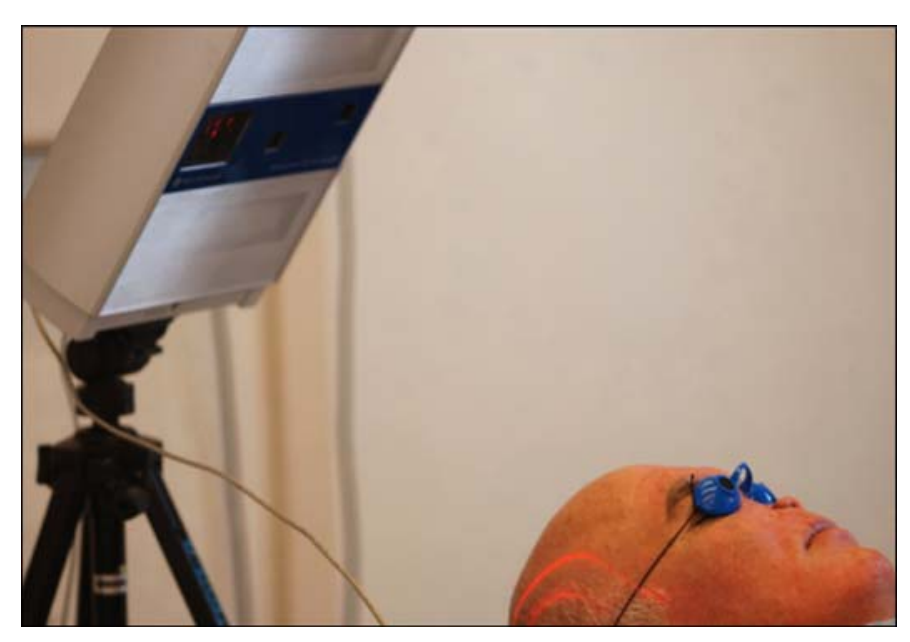

Figure 3.

Scanning of patient's injured face with three-dimensional laser (NextEngine Desktop 3-D Scanner, NextEngine, Inc; Santa Monica, California).

acquisition, the patient was asked to keep his head firmly positioned in the headrest and not to smile, which kept the maxillary arches closed in the maximal intercuspal position. To obtain a good result and simplify the later alignment of the different scans during the postprocessing operations, the clinician must ensure that the patient avoids any movements during the scanning process, as well as any alterations of the external profile of the face (i.e., smiling or contracting areas).

Then, we digitized a pair of eyeglasses chosen by the patient using a Konica Minolta VIVID 9i laser scanner (Konica Minolta Sensing, Inc; Osaka, Japan). First, the eyeglasses were coated with talcum powder, with the temple arms opened to avoid any problems during the scan process due to the transparency of the lens and the highly reflective material of the frame. To obtain the complete coverage of external and internal surfaces of the eyeglasses, we needed 20 scans.

As is usual in reverse engineering, we postprocessed both data sets (patient's face and eyeglasses) to obtain the final digital models in solid-to-layer format. We first aligned the scans by identifying three pairs of morphological reference points on the overlapping regions of the two corresponding different scans. After we aligned all the scans, we refined the alignment semiautomatically using a fine registration and, finally, merged all scans together. Additional finishing operations were performed for eliminating any surface abnormalities, followed by remeshing and smoothing the two triangular meshes.
For both data sets, we performed all postprocessing operations needed to align the scans, merge the scans, and finish the models (such as deleting abnormal faces, filling holes, remeshing, smoothing, etc.) with Rapidform XOS software (INUS Technology; Seoul, South Korea). Moreover, we checked the eyeglasses position using a puttysilicon (Elite HD, Zhermack; Badia Polesine, Italy) during the first appointment: the silicon was positioned in the glabella region and the eyeglasses were pressed on it until the correct position was gained. We also scanned the impressed putty to be integrated in the virtual environment, ensuring to reposition the eyeglasses in the same respective position as the real one. No direct scanning of the eyeglasses on the face was taken because, without the nose, their accurate and stable position may be customized only with a silicone engaging the skin and the eyeglasses at the same time.

Next, we designed the customized prosthesis with the support of an "Ear \& Nose Digital Library," which is used mainly when a contralateral healthy side of the patient's face is not available to develop a prosthesis using a mirroring-based approach, such as for an ear prosthesis. The Ear \& Nose Digital Library used in this work is based on real anatomic models collected during a hands-on study of maxillofacial prosthodontics. Stone models of auricular and nasal epitheses of students were digitized and stored with the necessary information (age, sex, and height) for creating a database of digital models. Starting from the 3-D model of the patient's face (Figure 4(a)), we chose a digital anatomic nose as a reference model from the digital library using earlier photographs of the patient, as well as his facial dimensions. We superimposed the digital model on the defect and positioned it in the correct anatomic location so that it covered the entire defect, adjusting the margin of the prosthesis to the patient's skin and designing the holes for the nostrils (Figure 4(b)). Once we designed the volume of the nasal prosthesis, we chose the thickness that reduced the mass of the prosthesis as much as possible, giving a minimum $6 \mathrm{~mm}$ backward offset of the prosthesis. Because the prosthesis supports the eyeglasses, it did not fill the volume of the defect, reducing the weight of the prosthesis. Moreover, a thin prosthesis is more easily adaptable to the patient's defect because of the elastic properties of the silicone.

Then, we placed the digital model of the eyeglasses on the 3-D model of the patient's face with reference to the existing anatomical structures previously scanned, as well as to the designed nasal epithesis (Figure 4(c)). In particular, the rims of the lenses were centered on the 
(a)

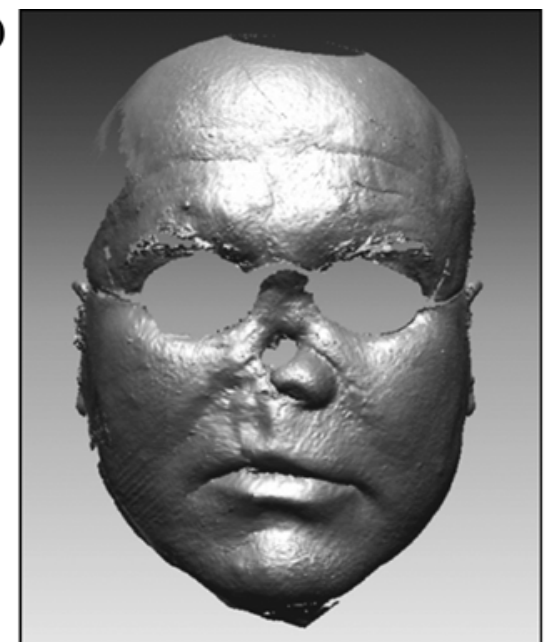

(b)

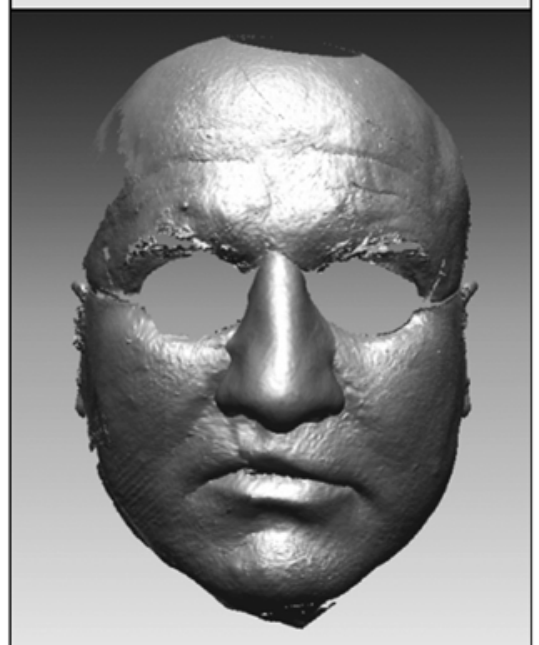

(c)

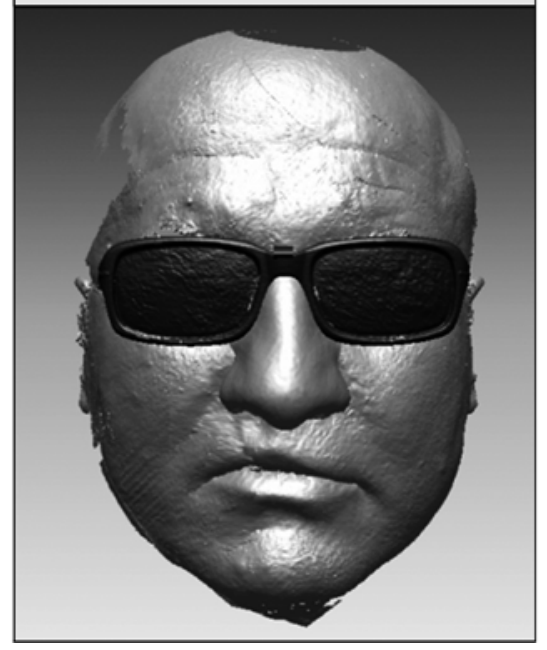

Figure 4.

(a) Three-dimensional model of patient's injured face, (b) superimposition of digitized reference nose on patient's face, and (c) digital model of eyeglasses positioned with reference to patient's eyes and ears with prosthesis. eyes, the temple arms were placed over the ears, and the bridge of the optical frame was put in contact with the upper part of the nasal prosthesis. The correct position of the eyeglasses was also checked by means of the digital model of the reference putty silicone.

After the digital models of the nasal prosthesis and eyeglasses were both positioned on the 3-D representation of the patient's face in Rapidform XOS, we imported them (with the new correct positions) in the CAD environment Rhino 4.0 (McNeel North America; Seattle, Washington) to design the connecting substructure and the mold for silicone processing.

We designed the substructure, accounting for volume constraints and functional requirements. We chose the length of the substructure $(55 \mathrm{~mm})$ according to the length of the prosthesis and designed the width $(8 \mathrm{~mm})$ and the thickness (from $3.5 \mathrm{~mm}$ in the upper part to $2.0 \mathrm{~mm}$ in the lower part), accounting for function so that the weight of the prosthesis and the dimensions of the bar clips were properly sustained. The substructure must be contained entirely within the silicone nasal prosthesis, except for the upper part, which is connected to the frame of the eyeglasses (Figure 5(a)). This upper part allows the technician to position the eyeglasses with respect to the substructure, and it was removed after the silicone was processed. We calculated the seat for the connection in a CAD environment by subtracting the volume of the bridge of the eyeglasses from the upper part of the substructure (Boolean volume difference). In this way, the substructure could be positioned precisely and unambiguously and fixed on the eyeglasses. Moreover, we added three rectangular holes to the main body of the substructure to guarantee mechanical fixation inside the silicone prosthesis (Figure 5(b)).

Finally, the nasal model was represented as a negative volume and split into two elements for obtaining a twopart mold for subsequent conventional silicone processing procedures (Figure 5(c)). In the upper part of the mold, a reference seat was designed for keeping the substructure in the planned position during silicone processing. When the silicone polymerized, the upper part of the substructure was eliminated, making it invisible behind the eyeglasses' interocular connector.

Next, the substructure and two-part mold were manufactured directly with the use of rapid prototyping techniques. A Stratasys Dimension soluble support technology 3-D printer (Stratasys; Eden Prairie, Minnesota) based on fused deposition modeling (FDM) was used for producing 
(a)

(b)

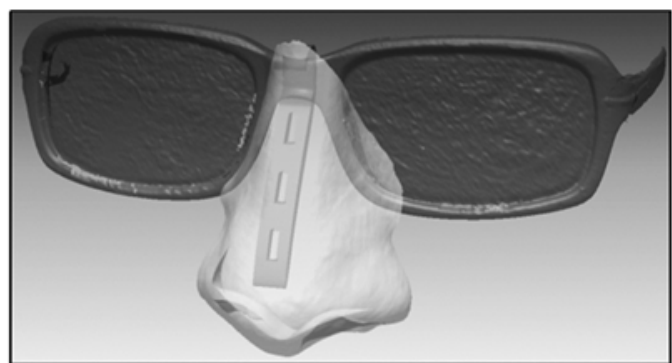

(c)
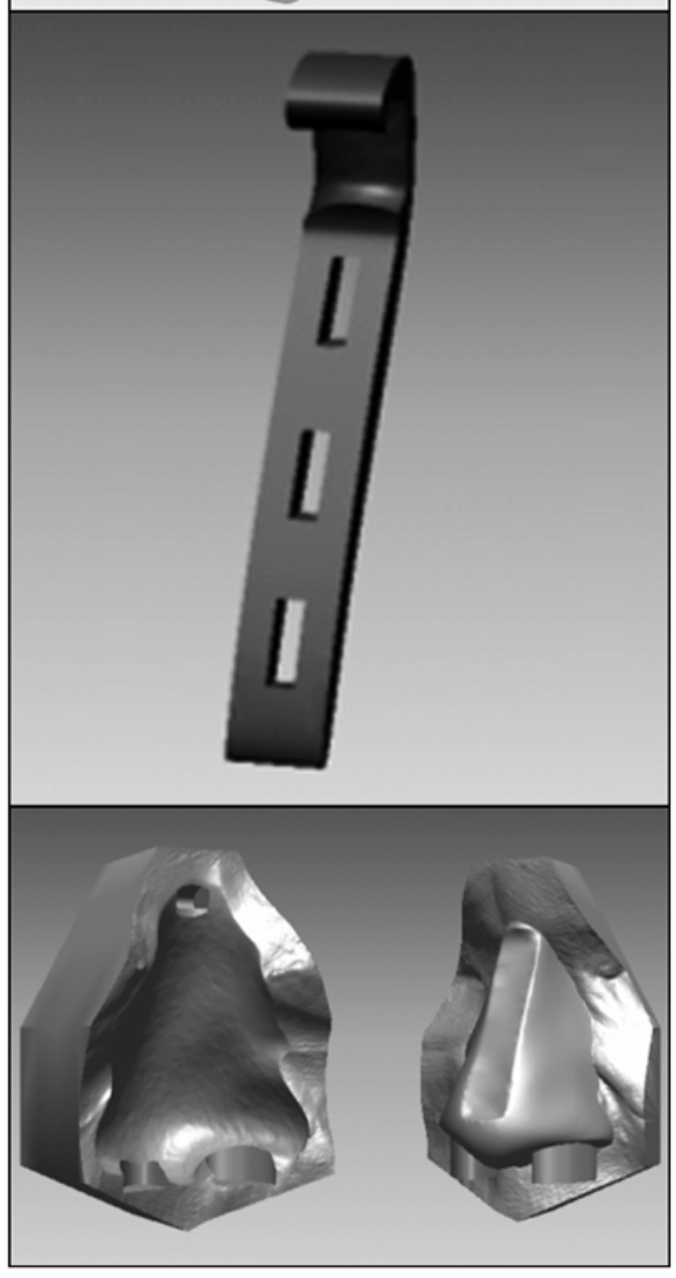

Figure 5.

(a) Digital model of prosthesis with substructure for connecting it to eyeglasses, (b) three-dimensional (3-D) model of substructure, and (c) 3-D model of two-part mold.

physical models in acrylonitrile butadiene styrene (ABS). The working principle of this system is based on layer-bylayer deposition with a layer thickness of $0.254 \mathrm{~mm}$ of both fused ABS and soluble support material for the sustaining of the overhanging parts of the component under construction. At the end of the building process, the model is placed in a hot soapy water bath, with agitation for the washing process to remove all the support material. Table 1 lists the ABS and support materials needed, building time, and cost for each model.

Before proceeding with the silicone molding procedures, we positioned the ABS substructure on the eyeglasses to check whether the planned connection was unambiguous and easily determined. Then, a hole was drilled in both the eyeglasses and substructure in the connecting region at the level of the eyeglasses. An implant abutment (Multi-Unit Abutment, external connection; NP, Nobel Biocare; Kloten, Switzerland) was secured in the eyeglasses with epoxy resin, and an implant replica (NP, Nobel Biocare) was inserted in the corresponding part of the substructure. The rigid connection to retain the nasal prosthesis was secured with a bolt (Figure 6(a)).

During the conventional silicone processing procedures, the substructure was disconnected from the eyeglasses and positioned in the mold with use of the reference hole in the upper part of the mold (Figure 6(b)) that was previously projected for simple repositioning of the substructure in the mold. We processed the silicone (VST-50F, Factor II; Lakeside, Arizona) to obtain a provisional nasal prosthesis using the ABS mold, taking care that silicone was deposited all around the substructure by using a syringe to inject the silicone (Figure 7(a)). Finally, we refined the prosthesis by trimming off the excess silicone at the margins.

According to the designed dimensions of the substructure and to the reduced thickness (and mass) of the silicone prosthesis, the FDM ABS material can ensure functional requirements in both strength and durability.

We used a spectrophotometer (SpectroShade Office; MHT SpA; Verona, Italy) to determine the intrinsic color of the prosthesis. The spectrophotometer used a color

Table 1.

Acrylonitrile butadiene styrene (ABS) and support materials, building time, and cost of rapid prototyping models.

\begin{tabular}{|c|c|c|c|c|}
\hline Portion & $\begin{array}{c}\text { ABS } \\
\text { Material } \\
\left(\mathrm{cm}^{3}\right)\end{array}$ & $\begin{array}{c}\text { Support } \\
\text { Material } \\
\left(\mathrm{cm}^{3}\right)\end{array}$ & $\begin{array}{l}\text { Building } \\
\text { Time }\end{array}$ & $\begin{array}{c}\text { Cost of } \\
\text { Material } \\
(€)^{*}\end{array}$ \\
\hline Mold Lower Part & 24.29 & 7.19 & $3 \mathrm{~h} 29 \mathrm{~min}$ & 8.70 \\
\hline Mold Upper Part & 38.18 & 11.36 & $5 \mathrm{~h} 12 \mathrm{~min}$ & 12.73 \\
\hline Substructure & 1.45 & 1.17 & $21 \mathrm{~min}$ & 2.27 \\
\hline Total Nose & 19.09 & 6.24 & 9 h 2 min & 23.70 \\
\hline
\end{tabular}




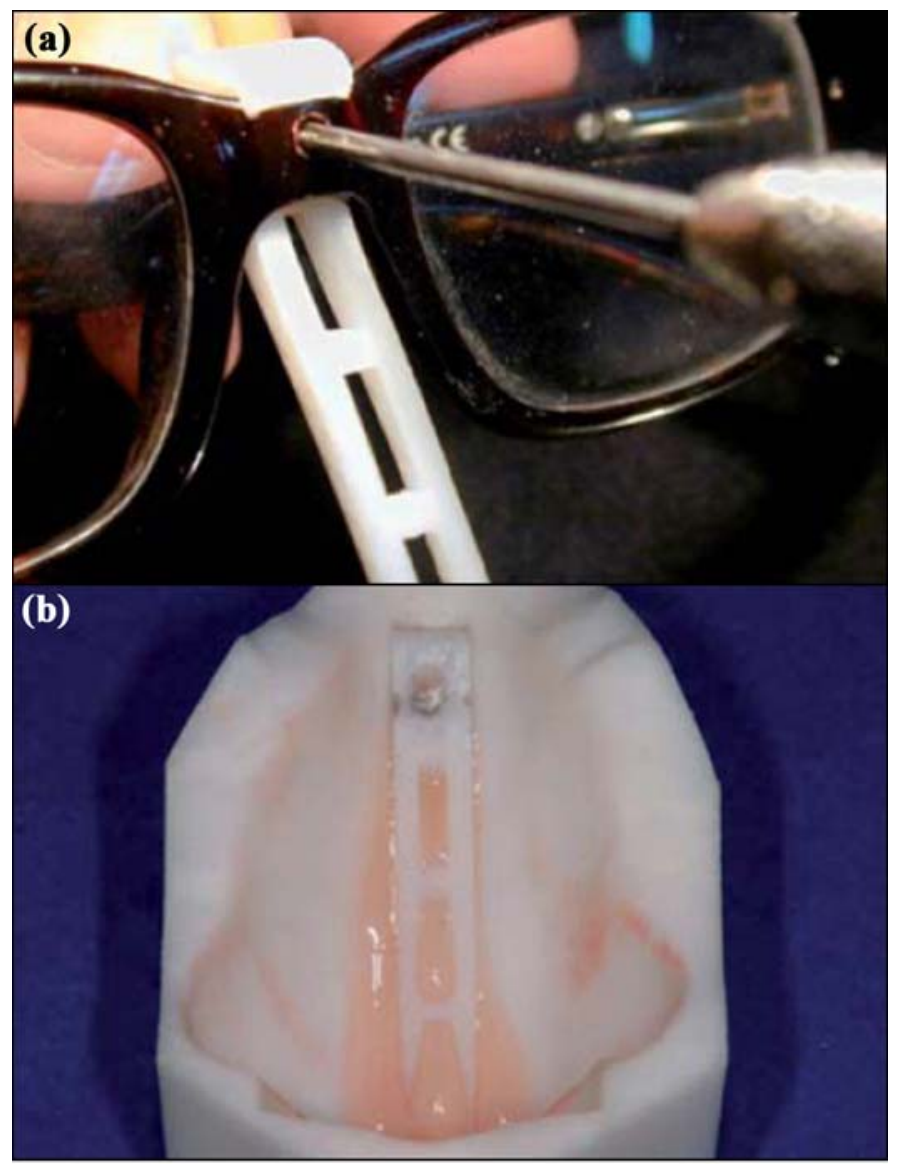

Figure 6.

(a) Connection used to retain nasal prosthesis consisting of a bolt passing through hole drilled in frame of eyeglasses and a nut inserted in upper part of substructure and (b) development of nasal epithesis using silicone process (positioned substructure is visible in mold).

grade elaborated by one of the authors: 12 silicone different colors were saved into the software of the machine. For obtaining each color, the mixture composition of the different intrinsic colors necessary was determined. When the patient had his first appointment, a registration of his skin and the prevalent intrinsic color was done with the use of the spectrophotometer. We used this color to pigment the silicone before molding. Extrinsic coloring (extrinsic, factor II) during the second (last) appointment was applied, silicone adhesive (A-564, factor II) was used as a sealant, and matting dispersion liquid (TS-564, factor II) was added for conferring a matte appearance to the final prosthesis. Finally, we reconnected the provisional prosthesis to the eyeglasses with a bolt (Figure 7(b)) and delivered the prosthesis to the patient (Figure 8).

\section{RESULTS}

The process was validated experimentally with in vivo application of the prosthesis on the patient. We observed two main results in terms of quality. First, the final prosthesis and virtually planned design were compared by a qualitative two-dimensional superimposition of a digital photograph of the patient, with the prosthesis in position and the corresponding view of digital model (Figure 9). To ensure a better and quantitative comparison, one could scan the patient's face with the prosthesis in position after delivering the prosthesis to analyze any surface deviation between the 3-D digital models of planned and final prosthesis. However, we believe that this kind of quantitative analysis would be more appropriate for evaluating the result of a definitive prosthesis retained by osseointegrated craniofacial implants. We also verified the position of the prosthesis as correct clinically by careful observation. The nasal prosthesis fit over the defect well, leaving no open margins in the contact region. Consequently, the patient did not have to use skin adhesive to retain it in the correct position.

Second, the connection between the prosthesis and eyeglasses was precise and unambiguous because of the Boolean volume difference calculated between the digital models. The design of the reference seat in the upper part of the mold ensured the precise positioning of the substructure in the mold. In addition, the mold resulted in a stable, secure position during silicone processing because of the design of the opposing parts. The holes designed in the main body of the substructure securely retained the substructure in the silicone prosthesis.

\section{DISCUSSION}

The Ear \& Nose Digital Library can be used to choose a reference model based on the patient's anatomy when no presurgical model is available or mirroring (as for ear prosthesis) is impossible. However, when surgical ablation of a tumor is scheduled, laser scanning of the face anatomy should always be performed before the ablative surgery. In this way, the digital model of the presurgical anatomy can be used to design the required prosthesis, even if the tumor alters the "model." One can incorporate all the necessary corrections in the virtual model to design the final correct anatomy. 
(a)

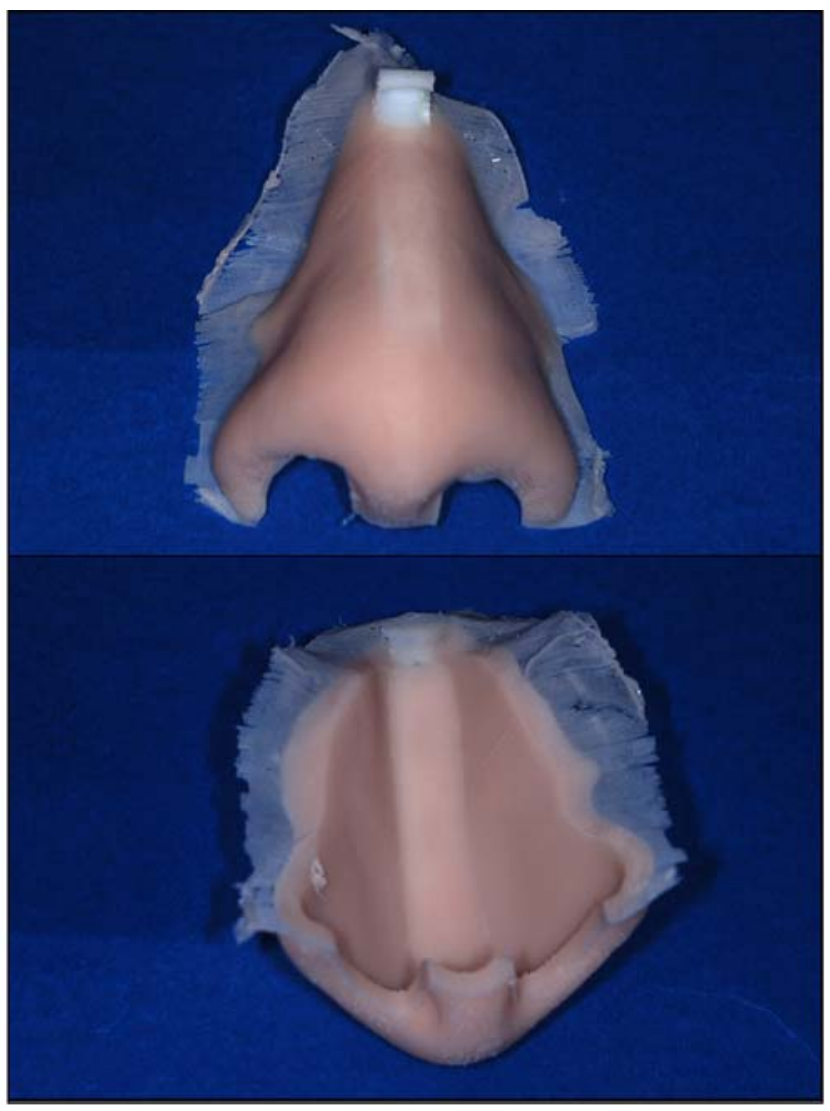

(b)

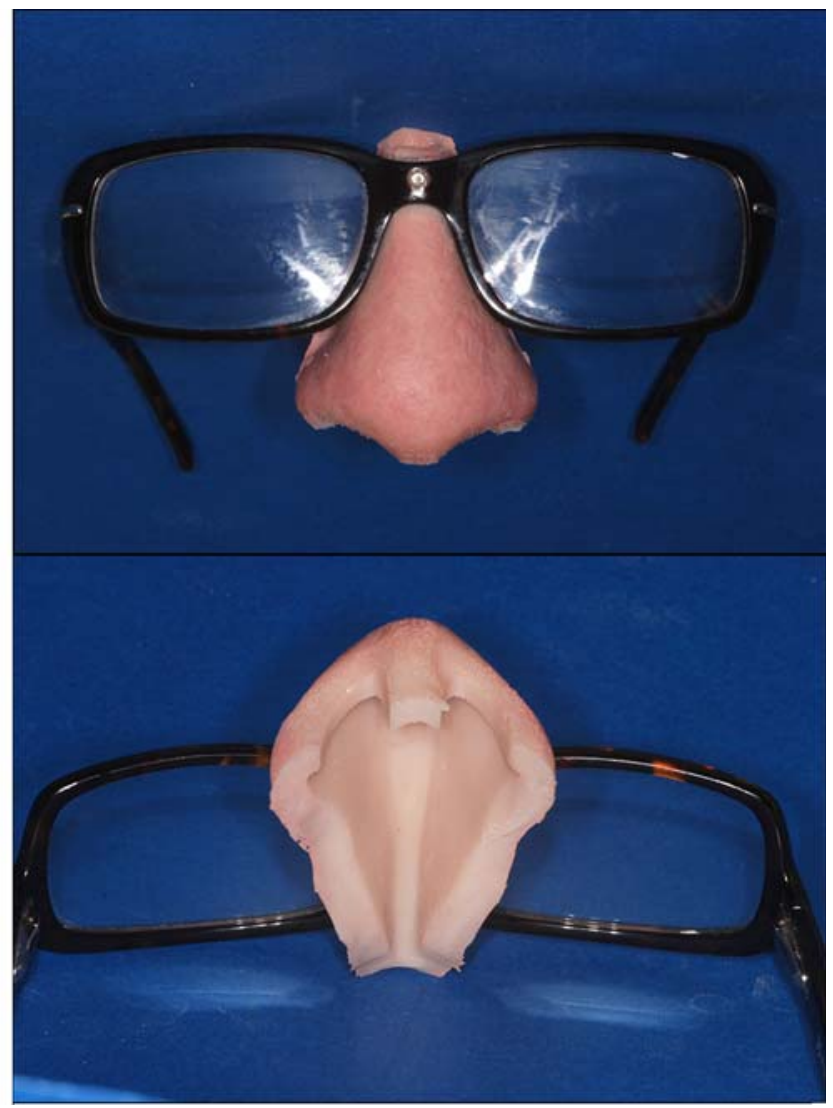

Figure 7.

Silicone prosthesis (a) before and (b) after finishing operations and connection to eyeglasses.

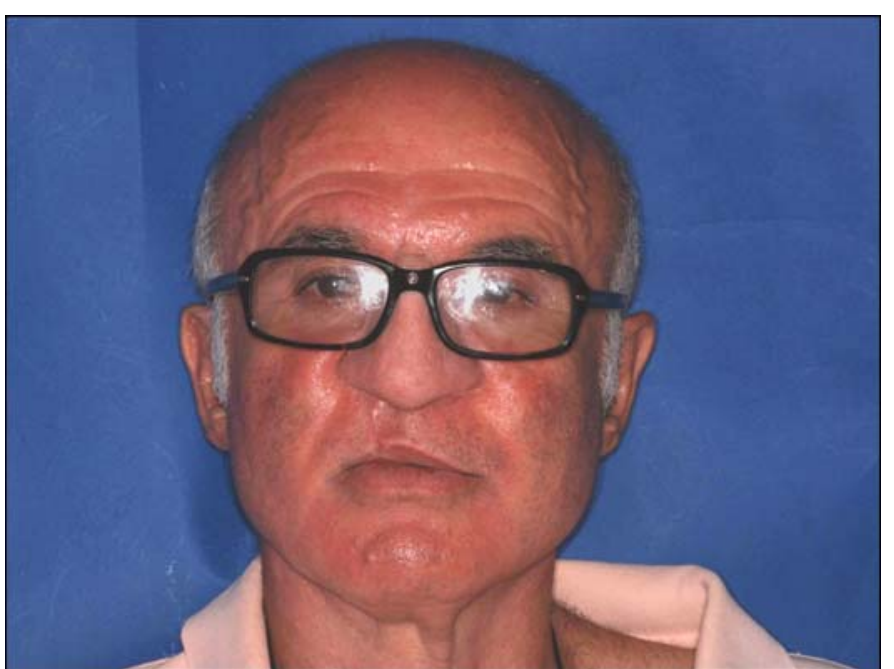

Figure 8.

Final prosthesis on patient.
However, given the required static position of the head for acquisition of the scans, we considered some practical aspects to ensure the prosthesis fitted well onto an animated face in vivo. First, the reference regions of the prosthesis were chosen according to stable structures only, e.g., as glabella and skin/bone borders of the defect. Second, since the external margins of the prosthesis rest against the mobile cut of the face, a thin overlapping margin of silicone was CAD-designed that may be flexible as the mimic movements require. In this way, the prosthesis can be stable and no margin is visible during functional movements of the face.

A problem might be the retention of the ABSsubstructure into the silicone. In our opinion, since no evidence-based data exist in the literature (to our knowledge) about the retentive forces of adhesion between the silicone and the ABS or polymethyl methacrylate (acrylic), the retention of the substructure has to be 

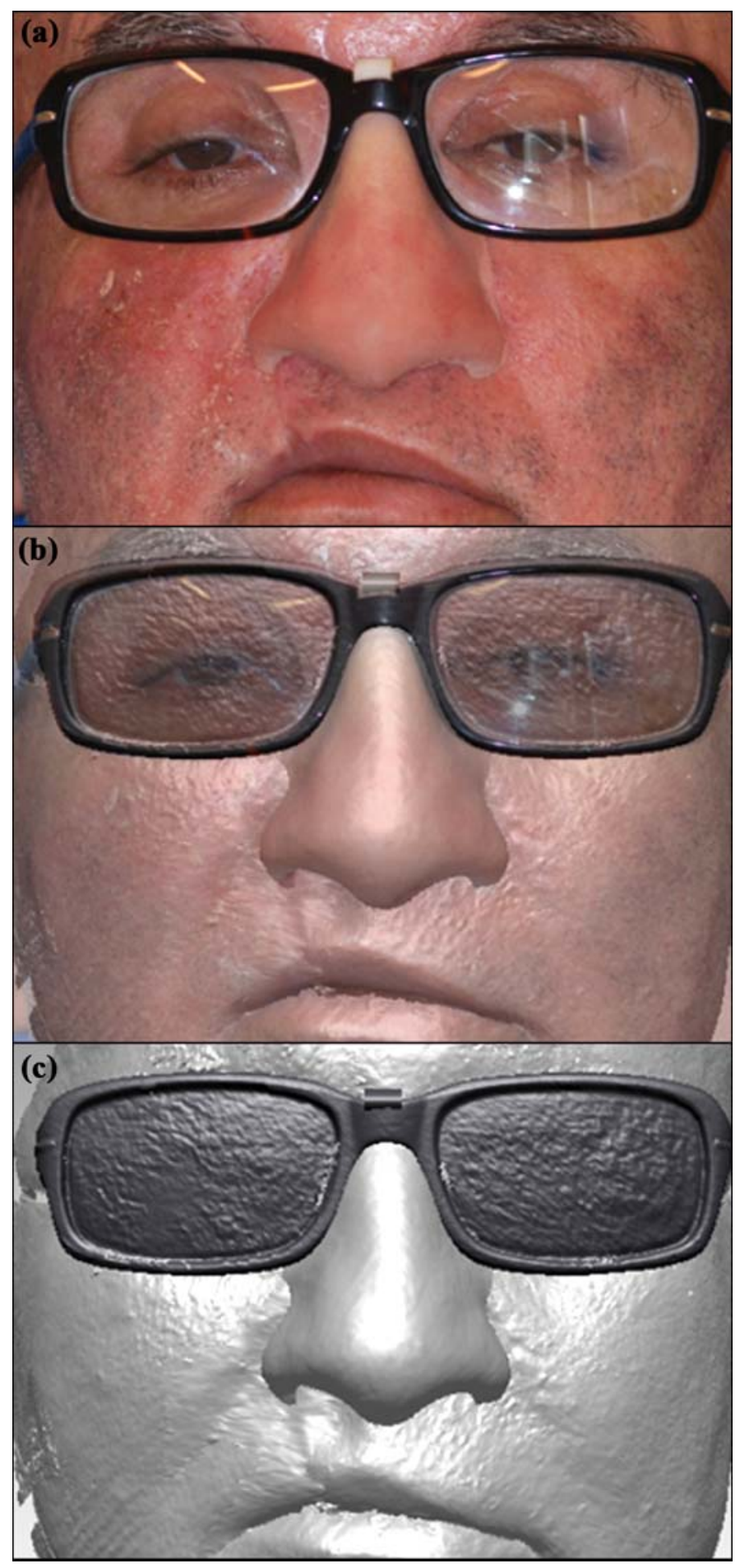

Figure 9.

Comparison of (a) final prosthesis and (b) and (c) virtually planned design.

mechanical and not only adhesive. For this reason, also in the case presented here, a series of connected holes is designed into the substructure that allows penetrating the silicone and engaging the substructure safely. The design of the holes has to compensate for mechanical properties of the substructure and retentive forces of silicone so that repositioning or removing the prosthesis does not dislodge the substructure from the silicone.

The main innovation of our protocol involves the digitization of the eyeglasses. The digitization of the eyeglasses allows the design of the connection between the digital models of the eyeglasses and the substructure of the nasal prosthesis in a virtual environment and enables an evaluation of the result on the 3-D model of the patient's face. The relative positions of the substructure and eyeglasses must be ensured with high accuracy, because an error at this stage would compromise the entire work, affecting the final prosthesis. Moreover, we transferred the eyeglasses position to the CAD designer using a putty silicone during the first appointment to ensure that the reposition of the eyeglasses in the virtual environment is in the same as the real world.

Compared with published procedures, our multidisciplinary protocol involving laser scanning, CAD/CAM, and rapid prototyping technologies for manufacturing a provisional nasal prosthesis introduces three improvements. First, our procedure shortens the time required to manufacture the provisional prosthesis, because only two appointments are necessary before the prosthesis is delivered: one to make a digital impression of the patient's face, including a skin color scan (for intrinsic coloring), and another for extrinsic coloring, try-in, and delivering the prosthesis. The total clinical time employed to perform these procedures is 3 hours and 40 minutes. In addition to this time, an additional 27 hours (of which, the operator does not need to be present for 19 hours) are needed for completing the procedure (Table 2) according to each step of the flow chart in Figure 2.

Second, because of the improvements of this protocol, our method is less expensive. The cost comparison between the conventional manual procedures (€811) and the innovative CAD/CAM protocol (€413) is presented in Table 3. A 50 percent cost savings can be seen.

Third, patient discomfort is reduced because the virtually planned position of the digital models of eyeglasses and nose eliminates the need for a conventional try-in appointment involving the placement of silicone on the patient's face and a nasal prosthesis wax-up. Reducing patient discomfort means improving patient quality of life.

Finally, the digital model of the designed nasal epithesis can be used to develop a definitive prosthesis anchored to osseointegrated craniofacial implants. Therefore, in terms of quality of care, scanning the eyeglasses and transferring 
Table 2.

Time needed to complete eyeglasses-supported prosthesis procedure.

\begin{tabular}{|c|c|c|c|}
\hline Step & $\begin{array}{c}\text { With } \\
\text { Patients }\end{array}$ & $\begin{array}{l}\text { Without } \\
\text { Patients }\end{array}$ & Total \\
\hline \multicolumn{4}{|l|}{ Reverse Engineering } \\
\hline $\begin{array}{l}\text { 3-D Laser Scanning of } \\
\text { Face }\end{array}$ & $40 \min$ & - & $40 \min$ \\
\hline $\begin{array}{l}\text { 3-D Laser Scanning of } \\
\text { Eyeglasses }\end{array}$ & - & $1 \mathrm{~h}$ & $1 \mathrm{~h}$ \\
\hline Computer-Aided Design & - & $6 \mathrm{~h}$ & $6 \mathrm{~h}$ \\
\hline \multicolumn{4}{|l|}{ Rapid Prototyping } \\
\hline Building Time & - & $9 \mathrm{~h}^{*}$ & $9 \mathrm{~h}^{*}$ \\
\hline Washing Time & - & $6 \mathrm{~h}^{*}$ & $6 \mathrm{~h}^{*}$ \\
\hline Connection Set-Up & - & $20 \min$ & $20 \min$ \\
\hline \multicolumn{4}{|l|}{ Silicone Processing } \\
\hline Injection & - & $20 \min$ & $20 \mathrm{~min}$ \\
\hline Hardening & - & $4 \mathrm{~h}^{*}$ & $4 \mathrm{~h}^{*}$ \\
\hline Screw Connection & - & $20 \min$ & $20 \min$ \\
\hline Extrinsic Coloring & $3 \mathrm{~h}$ & - & $3 \mathrm{~h}$ \\
\hline Total & $3 \mathrm{~h}, 40 \mathrm{~min}$ & $27 \mathrm{~h}$ & $30 \mathrm{~h}, 40 \mathrm{~min}$ \\
\hline
\end{tabular}

Table 3.

Cost comparison between computer-aided design/computer-aided manufacturing (CAD/CAM) and manual procedure.

\begin{tabular}{|c|c|c|c|c|}
\hline Variable & $\begin{array}{c}\text { Cost of } \\
\text { CAD/CAM } \\
\text { Procedure } \\
\text { (€) }\end{array}$ & Notes & $\begin{array}{c}\text { Cost of } \\
\text { Manual } \\
\text { Procedure } \\
(€)\end{array}$ & Notes \\
\hline$\overline{\mathrm{CAD}}$ & 250 & - & - & - \\
\hline \multicolumn{5}{|l|}{ Designer } \\
\hline Substructure & 12 & $\begin{array}{l}\text { Manufactured } \\
\text { by rapid } \\
\text { prototyping }\end{array}$ & 120 & $\begin{array}{l}\text { Manufactured } \\
\text { by technician }\end{array}$ \\
\hline Mold & 60 & $\begin{array}{l}\text { Manufactured } \\
\text { by rapid } \\
\text { prototyping }\end{array}$ & 600 & $\begin{array}{l}\text { Manufactured } \\
\text { by } \\
\text { anaplastologist }\end{array}$ \\
\hline Silicone & 4 & - & 4 & - \\
\hline $\begin{array}{l}\text { Connection } \\
\text { System }\end{array}$ & 87 & - & 87 & - \\
\hline Total & 413 & - & 811 & - \\
\hline
\end{tabular}

their position from the patient straight into the virtual environment considerably increases precision and reduces the patient's time investment.

\section{CONCLUSIONS}

A new protocol for making an immediate provisional eyeglasses-supported nasal prosthesis is presented that uses laser scanning, $\mathrm{CAD} / \mathrm{CAM}$ procedures, and rapid prototyping techniques. Compared with existing methods, our protocol reduces time and costs and restores the patient's aesthetic appearance after the disfiguration caused by ablation of the nasal pyramid.

\section{ACKNOWLEDGMENTS}

\author{
Author Contributions: \\ Study concept and design: L. Ciocca. \\ Acquisition of data: M. Fantini, F. Decrescenzio. \\ Analysis and interpretation of data: L. Ciocca, M. Fantini. \\ Drafting of manuscript: L. Ciocca, M. Fantini. \\ Critical revision of manuscript for important intellectual content: \\ R. Scotti, F. Persiani. \\ Study Supervision: L. Ciocca.
}

Financial Disclosures: The authors have declared that no competing interests exist.

Funding/Support: This material is the result of work supported with resources and the use of facilities at the II Faculty of Engineering, University of Bologna, Forli, Italy.

Additional Contributions: The English in this document has been checked by at least two editors, both professional English translators. For a certificate, please see http://www.textcheck.com/certificate/ $\underline{\text { TLfiwU/. }}$.

Participant Follow-Up: The authors plan to inform participant of the publication of this study.

\section{REFERENCES}

1. Beumer J, Curtis TA, Marunick MT. Maxillofacial rehabilitation: Prosthodontic and surgical considerations. St. Louis (MO): Medico Dental Media International, Inc.; 1996. p. 377-453.

2. Javid $N$. The use of magnets in a maxillofacial prosthesis. J Prosthet Dent. 1971;25(3):334-41. [PMID: 5276856] DOI:10.1016/0022-3913(71)90196-X

3. Ugadama A, King GE. Mechanically retained facial prostheses: Helpful or harmful? J Prosthet Dent. 1983;49(1): 85-86. [PMID: 6571903] DOI:10.1016/0022-3913(83)90245-7

4. Parel SM, Branemark P-I, Tjellstrom A, Gion G. Osseointegration in maxillofacial prosthetics. Part II: Extraoral applications. J Prosthet Dent. 1986;55(5):600-606.

[PMID: 3458911]

DOI:10.1016/0022-3913(86)90040-5

5. Dumbrigue HB, Fyler A. Minimizing prosthesis movement in a midfacial defect: A clinical report. J Prosthet Dent. 1997;78(4):341-45. [PMID: 9338864] DOI:10.1016/S0022-3913(97)70040-4 
6. Flood TR, Russell K. Reconstruction of nasal defects with implant-retained nasal prostheses. $\mathrm{Br} \mathrm{J}$ Oral Maxillofac Surg. 1998;36(5):341-45. [PMID: 9831053]

DOI:10.1016/S0266-4356(98)90644-1

7. Cheah CM, Chua CK, Tan KH, Teo CK. Integration of laser surface digitizing with CAD/CAM techniques for developing facial prostheses. Part 1: Design and fabrication of prostheses replicas. Int J Prosthodont. 2003;16(4):435-41. [PMID: 12956501]

8. Cheah CM, Chua CK, Tan KH. Integration of laser surface digitizing with CAD/CAM techniques for developing facial prostheses. Part 2: Development of molding techniques for casting prosthetic parts. Int J Prosthodont. 2003; 16(5):543-48. [PMID: 14651243]

9. Ciocca L, Scotti R. CAD-CAM generated ear cast by means of a laser scanner and rapid prototyping machine. J Prosthet Dent. 2004;92(6):591-95. [PMID: 15583570] DOI:10.1016/j.prosdent.2004.08.021

10. Ciocca L, Mingucci R, Gassino G, Scotti R. CAD/CAM ear model and virtual construction of the mold. J Prosthet Dent. 2007;98(5):339-43. [PMID: 18021820] DOI:10.1016/S0022-3913(07)60116-4

11. Jiao T, Zhang F, Huang X, Wang C. Design and fabrication of auricular prostheses by CAD/CAM system. Int J Prosthodont. 2004;17(4):460-63. [PMID: 15382783]

12. Feng Z, Dong Y, Zhao Y, Bai S, Zhou B, Bi Y, Wu G. Computer-assisted technique for the design and manufacture of realistic facial prostheses. Br J Oral Maxillofac Surg, 2010; 48(2):105-9. [PMID: 19556041]

DOI:10.1016/j.bjoms.2009.05.009

13. Ciocca L, De Crescenzio F, Fantini M, Scotti R. CAD/CAM bilateral ear prostheses construction for Treacher Collins syndrome patients using laser scanning and rapid prototyping. Comput Methods Biomech Biomed Engin. 2010;13(3): 379-86. [PMID: 19844817]

DOI:10.1080/10255840903251304

14. Ciocca L, Maremonti P, Bianchi B, Scotti R. Maxillofacial rehabilitation after rhinectomy using two different treatment options: Clinical reports. J Oral Rehabil. 2007;34(4): 31115. [PMID: 17371570$]$ DOI:10.1111/j.1365-2842.2007.01684.x

15. Ciocca L, Bacci G, Mingucci R, Scotti R. CAD-CAM construction of a provisional nasal prosthesis after ablative tumour surgery of the nose: A pilot case report. Eur J Cancer Care (Engl). 2009;18(1):97-101. [PMID: 19473226] DOI:10.1111/j.1365-2354.2008.01013.x

16. Ciocca L, Fantini M, Marchetti C, Scotti R, Monaco C. Immediate facial rehabilitation in cancer patients using CAD-CAM and rapid prototyping technology: A pilot study. Support Care Cancer. 2010;18(6):723-28. [PMID: 19513762] DOI:10.1007/s00520-009-0676-5

Submitted for publication November 26, 2009. Accepted in revised form May 7, 2010.

This article and any supplementary material should be cited as follows:

Ciocca L, Fantini M, De Crescenzio F, Persiani F, Scotti R. New protocol for construction of eyeglasses-supported provisional nasal prosthesis using CAD/CAM techniques. J Rehabil Res Dev. 2010;47(7): 595-04.

DOI:10.1682/JRRD.2009.11.0189 